Revue de droit comparé du travail et de la sécurité sociale

3 | 2017

Le travail dans l'économie informelle, un défi pour le droit social

\title{
Résiliation du contrat de travail pour des motifs liés au comportement du salarié
}

Ljubinka Kovačević

\section{CpenEdition}

Journals

Édition électronique

URL : https://journals.openedition.org/rdctss/461

DOI : $10.4000 /$ rdctss.461

ISSN : 2262-9815

Éditeur

Centre de droit comparé du travail et de la sécurité sociale

Édition imprimée

Date de publication : 1 septembre 2017

Pagination : 226-229

ISSN : $2117-4350$

Référence électronique

Ljubinka Kovačević, «Résiliation du contrat de travail pour des motifs liés au comportement du salarié », Revue de droit comparé du travail et de la sécurité sociale [En ligne], 3 | 2017, mis en ligne le 01 novembre 2017, consulté le 04 décembre 2021. URL : http://journals.openedition.org/rdctss/461 ; DOI : https://doi.org/10.4000/rdctss.461

\section{(c) (i) (9)}

Revue de droit comparé du travail et de la sécurité sociale est mise à disposition selon les termes de la Licence Creative Commons Attribution - Pas d'Utilisation Commerciale - Pas de Modification 4.0 International. 


\section{LJUBINKA KOVAČEVIĆ Université de Belgrade}

\section{RÉSILIATION DU CONTRAT DE TRAVAIL POUR DES MOTIFS LIÉS AU COMPORTEMENT DU SALARIÉ}

Loi sur le travail (Journal Officiel RS, no. 24/05), article 179 , alinéa 4.

Dans le droit de la République de Serbie, le comportement fautif du salarié peut avoir les conséquences suivantes : a) la résiliation du contrat de travail pour un motif justifié du fait que le salarié a violé ses obligations, a manqué à la discipline du travail, ou a été reconnu coupable par une décision judiciaire d'une infraction pénale commise au travail et en rapport avec le travail ; b) la cessation ex lege de la relation de travail en raison de l'absence du salarié durant plus de six mois en exécution d'une sanction pénale ; cl l'éloignement du salarié de son poste de travail soit en cas de détention provisoire, soit en cas de procédure pénale suite à une infraction pénale; d) la responsabilité du salarié du préjudice qu'il a causé dans le cadre de ses relations de travail.

La première des conséquences du comportement du salarié consiste plus précisément en la possibilité pour l'employeur d'en éliminer les effets néfastes en usant de sa faculté de résilier le contrat de travail pour un motif valable. Cependant, il revient à l'employeur d'estimer si les conditions d'un licenciement sont remplies et de mettre fin à une relation de travail par une déclaration de volonté. Toutefois, le risque d'abus est assez important en raison du manque de précision des dispositions légales en vigueur régissant certains motifs de licenciement. La garantie légale contre le licenciement injustifié peut ainsi être privée de contenu juridique mais aussi être éloignée du but pour lequel elle a été établie, comme en témoignent les récentes modifications apportées à la Loi sur le travail serbe dans la partie relative à la résiliation du contrat de travail en raison du comportement du salarié. L'une de ces modifications porte sur le licenciement pour infraction pénale dans le cadre des relations de travail, tandis qu'une autre modification résulte d'une décision de la Cour constitutionnelle de Serbie relative au licenciement pour manquement à la discipline du travail.

Le salarié peut commettre des infractions pénales dans le cadre du travail. Il peut s'agir d'infractions contre les biens, les biens personnels et les droits de l'employeur, des salariés et des tiers. Il s'agit d'infractions pénales définies par le Code pénal que le salarié peut commettre en effectuant des tâches qui lui sont confiées, comme la falsification d'un document officiel, la divulgation d'un secret, le détournement de fonds, la réception de pots-de-vin ou le vol. Ces infractions constituent un motif justifié de licenciement si et seulement si le tribunal a établi la responsabilité du salarié pour l'infraction commise. À cet égard, il convient de garder à l'esprit que le texte original de la Loi sur le travail de 2005 a confirmé le droit de l'employeur de résilier le contrat de travail, si le salarié commet une infraction pénale au travail ou en rapport avec le travail ${ }^{1}$. Certains employeurs ont interprété cette disposition légale comme une occasion de licencier le salarié avant même qu'il ne soit reconnu coupable de l'infraction pénale, alors que dans la littérature juridique il n'existait aucune opinion unanime sur les conditions d'application de ce motif de résiliation. Certains auteurs trouvaient que le licenciement pour ce motif devait être considéré indépendamment 
de la procédure pénale et que l'employeur pouvait licencier le salarié s'il apprenait que ce dernier avait commis une infraction pénale, s'il avait porté plainte contre le salarié et s'il existait des preuves sérieuses². D'autres, majoritaires, mettaient en garde contre le manque de précision de cette disposition légale et son caractère incomplet mais aussi contre les risques engendrés par son application, tout d'abord en ce qui concerne la mise en danger des droits de l'homme du salarié ${ }^{3}$. Il en allait de même pour la Cour suprême de Serbie dont la jurisprudence affirmait que « seule une décision pénale définitive de condamnation était constitutive d'un motif de licenciement et permettait alors à l'employeur de recourir au licenciement $»^{4}$. Cette interprétation a été confirmée explicitement par l'amendement de la Loi sur le travail adoptée en $2014^{5}$ qui a formellement mis un terme aux interprétations erronées dudit motif de licenciement.

En plus de l'infraction pénale comme motif de licenciement, la Loi sur le travail serbe visait un autre motif concernant le comportement du salarié et relatif à l'infraction pénale. Il s'agissait du pouvoir de l'employeur de résilier le contrat de travail du salarié qui ne respectait pas la discipline du travail, si son comportement représentait une infraction pénale dans le cadre de ses relations de travail ${ }^{6}$. Ce motif avait été confirmé comme un des motifs de licenciement pour manquement à la discipline du travail, de sorte que l'employeur pouvait estimer par lui-même si le comportement pouvait être qualifié d'infraction pénale ainsi que licencier le salarié pour cette raison (et non pour l'infraction pénale commise) s'il constatait que le comportement était constitutif d'une infraction pénale. À cet égard, il faut garder à l'esprit qu'en République de Serbie, la responsabilité disciplinaire des salariés dans le secteur privé est réglementée dans le cadre de la résiliation du contrat de travail, alors que d'autres mesures disciplinaires sont considérées comme des mesures pouvant être prononcées à la place du licenciement. Une liste indicative des violations des obligations du travail et des manquements à la discipline du travail pouvant donner lieu à licenciement figure dans la Loi sur le travail. Ces cas peuvent aussi être précisés dans les sources du droit autonome en fonction des spécificités de l'activité de l'entreprise et des particularités de l'environnement de travail. L'employeur peut utiliser ce motif de licenciement seulement si cette violation est préalablement établie comme une faute par le contrat de travail, la convention collective ou le règlement de travail. D'un autre côté, le manquement à la discipline du travail constitue une violation de n'importe 
Décision de la Cour suprême, Rev. 2406, du 13 juillet 2006.

8 Constitution de la République de Serbie (Journal Officiel RS, no. 98/06), articles 32 et 34.

Constitution de la République de Serbie (Journal Officiel RS, no. 98/06), article 21, paragraph 3.

10 Constitution de la République de Serbie (Journal Officiel RS, no. 98/06), articles 1 et 3.

11 Loi sur la Cour constitutionnelle IJournal Officiel $R S$, no. 109/07, 99/11, 18/13, 103, 15 et $40 / 15$ ), article 61. quelle obligation (principale ou secondaire) du salarié dans le cadre des relations de travail, il n'est donc pas nécessaire qu'elle soit établie comme un comportement punissable par les sources du droit autonome. Pour que ce motif de licenciement existe, il suffit que le comportement du salarié ne soit pas conforme aux exigences des actes réglementaires de l'employeur car tout manquement à la discipline peut donner lieu à licenciement si le comportement du salarié est tel qu'il rend impossible le maintien de la relation de travail?.

Conformément aux dispositions de la Loi sur le travail, l'employeur peut donc recourir au licenciement pour sanctionner le salarié quand son comportement « est une infraction pénale au travail ou en rapport avec le travail indépendamment du fait que les poursuites pénales aient été ou non engagées $\gg$. À cet égard, la Cour constitutionnelle de la République de Serbie a été saisie pour savoir si l'employeur a le droit de déterminer lui-même si le comportement du salarié représente une infraction pénale ou non.

La Cour constitutionnelle a considéré, à juste titre, que la disposition litigieuse du droit du travail n'était pas conforme à la Constitution étant donné que l'employeur avait un pouvoir dont seul pouvait disposer le tribunal lle pouvoir d'examiner si un acte ou une omission constitue une infraction pénale), alors que les salariés étaient privés de l'exercice des droits garantis. Il s'agit tout d'abord du droit à un procès équitable ainsi que du droit à la sécurité juridique consacré par le droit pénal qui suppose aussi la présomption d'innocence ${ }^{8}$. En outre, la Cour a considéré que ladite disposition était contraire au principe d'égalité de tous devant la Constitution et la loi'. La Cour a également confirmé la violation du principe de l'État de droit ${ }^{10}$ selon lequel les normes juridiques doivent être suffisamment claires, précises et prévisibles. Ainsi, la disposition contestée ne pouvait pas être qualifiée de cette manière, vu que l'employeur examinait lui-même l'existence d'un motif de licenciement sans avoir déterminé préalablement le comportement illégal du salarié si bien que les salariés ignoraient quels comportements l'employeur considèrerait comme des manquements à la discipline du travail et, de ce fait, ne pouvaient pas adapter leur comportement à la Loi ni être conscients des actes proscrits.

Une fois la décision rendue par la Cour constitutionnelle la disposition jugée inconstitutionnelle de la Loi sur le travail a cessé d'être appliquée. Les salariés licenciés en vertu de cette disposition ont eu le droit de demander à l'employeur de modifier la décision de licenciement rendue au nom de la disposition légale inconstitutionnelle dans un délai de six mois après la publication de la Décision de la Cour constitutionnelle (soit jusqu'au 4 août 2017) ${ }^{11}$, à condition que la décision de licenciement ait été prise 
au maximum deux ans avant la date de publication de la décision. Si l'employeur refuse de modifier la décision de licenciement, le salarié a le droit de demander devant le tribunal l'annulation de la décision de licenciement et la réparation du préjudice ainsi subi. Au cas où le tribunal rejette la demande, le salarié a le droit de déposer une plainte devant la Cour constitutionnelle à la suite de laquelle la Cour peut demander la restitution, la réparation du préjudice ou l'élimination des conséquences d'une autre manière. La question des procédures judiciaires engagées en raison du « comportement du salarié constitutif d'une infraction pénale » avant la publication de la décision de la Cour constitutionnelle reste ouverte en pratique. Cela est dû au fait que certains tribunaux affirme qu'au cours d'une procédure, un juge peut estimer si le comportement du salarié peut ou non correspondre à un autre motif de licenciement. Mais étant donné qu'il ne s'agit pas d'une opinion partagée par tous les tribunaux, il n'est pas certain que les juges se lancent dans ce type d'analyse du comportement du salarié en cas de résiliation du contrat de travail en vertu de la disposition légale déclarée.

Enfin, il faut souligner que la relation entre les motifs de licenciement liés à une infraction pénale et le manquement à la discipline du travail devient particulièrement délicate dans le cas où l'employeur a écarté de son poste de travail le salarié contre lequel a été engagée la procédure pénale. C'est l'hypothèse d'une mesure temporaire de mise à pied du salarié avec maintien de la rémunération jusqu'à ce que la responsabilité du salarié soit établie, pendant trois mois au maximum. Jusqu'à l'entrée en vigueur de la dernière modification de la Loi sur le travail, l'employeur avait deux possibilités avant l'expiration de la mesure prise : soit entamer la procédure de licenciement, soit faire revenir le salarié au travail. Or, dans la plupart des cas, la procédure pénale contre le salarié ne donne pas lieu à une décision définitive dans les trois mois, si bien que l'employeur ne pouvait pas licencier pour infraction pénale mais devait permettre la reprise du travail. Comme dans certains cas, ceci a entraîné des perturbations de l'environnement de travail, les employeurs essayaient de préserver le bon fonctionnement de l'entreprise en licenciant le salarié pour d'autres motifs justifiés. C'est tout d'abord le cas de la violation de l'obligation de travail, à condition que les éléments de la violation puissent être trouvés dans les agissements du salarié. Ces problèmes ont été résolus grâce à une nouvelle solution légale selon laquelle l'employeur est tenu de permettre la reprise du travail au salarié contre lequel des poursuites ont été engagées en raison d'une infraction pénale dans le cadre de la relation de travail, à l'expiration de la durée de la mise à pied ou de résilier le contrat de travail ou de prononcer tout autre mesure ${ }^{12}$. Un novum juridique correspond à la période de la mise à pied du salarié contre lequel des poursuites ont été engagées en raison d'une infraction pénale dans le cadre de la relation de travail qui désormais peut se poursuivre jusqu'à la décision définitive de la procédure pénale ${ }^{13}$. 